\title{
ORIGINAL ARTICLE Genetic factors influencing the risk of multiple myeloma bone disease
}

\author{
DC Johnson ${ }^{1}$, N Weinhold ${ }^{2,3}$, J Mitchell ${ }^{4}$, B Chen ${ }^{5}$, OW Stephens ${ }^{2}$, A Försti ${ }^{5,6}$, J Nickel ${ }^{3}$, M Kaiser ${ }^{1}$, WA Gregory ${ }^{7}$, D Cairns ${ }^{7}$, GH Jackson ${ }^{8}$, \\ P Hoffmann ${ }^{9,10}$, MM Noethen ${ }^{9,11}$, J Hillengass ${ }^{3}$, U Bertsch $^{3}$, B Barlogie ${ }^{2}$, FE Davis ${ }^{2}$, K Hemminki $^{5,6}$, H Goldschmidt ${ }^{3,12}$, RS Houlston ${ }^{1,4}$ and $^{3}$ \\ GJ Morgan ${ }^{2}$
}

A major complication of multiple myeloma (MM) is the development of osteolytic lesions, fractures and bone pain. To identify genetic variants influencing the development of MM bone disease (MBD), we analyzed MM patients of European ancestry (totaling 3774), which had been radiologically surveyed for MBD. Each patient had been genotyped for $~ 600000$ single-nucleotide polymorphisms with genotypes for six million common variants imputed using 1000 Genomes Project and UK10K as reference. We identified a locus at 8q24.12 for MBD ( $\mathrm{s} 4407910$, OPG/TNFRSF11B, odds ratio $=1.38, P=4.09 \times 10^{-9}$ ) and a promising association at $19 q 13.43$ ( $r$ 74676832, odds ratio $=1.97, P=9.33 \times 10^{-7}$ ). Our findings demonstrate that germline variation influences $M B D$ and highlights the importance of RANK/RANKL/OPG pathway in MBD development. These findings will contribute to the development of future strategies for prevention of MBD in the early precancerous phases of MM.

Leukemia (2016) 30, 883-888; doi:10.1038/leu.2015.342

\section{INTRODUCTION}

Multiple myeloma (MM) is a B-cell malignancy characterized by the expansion of clonal plasma cells in the bone marrow. ${ }^{1,2}$ The disease is typified by varying numbers of osteolytic lesions that are the result of reduced osteoblastic and increased osteoclastic activity. ${ }^{3,4}$ The presence of such bone lesions is a major criterion used to distinguish MM that requires treatment from precursor entities such as monoclonal gammopathy of undetermined significance and smoldering myeloma, which can be managed expectantly, ${ }^{5}$ as such, it is important to understand factors impacting on the development of MM bone disease (MBD). Although most patients develop osteoblastic lesions, a subset is unaffected by MBD, the reasons for which are not understood. Understanding the genetic mechanisms that are responsible for these differences in MBD is a pressing clinical issue, which has important implications for the development of novel treatments and in determining which patients might benefit from alternate bone therapies. In this respect, it has been shown in the setting of osteoporosis that heritable factors account for $50-85 \%$ of the variation in bone mineral density (BMD). ${ }^{6,7}$ As the interaction of bone-forming cells with MM cells is central to the development of an osteolytic lesion and MM clonal growth, we hypothesized that germline variation could also contribute to the development of MBD. We have recently conducted genome-wide association studies (GWAS) of MM searching for susceptibility alleles. ${ }^{8-10}$ Linking these genetic data to the extent of MDB at baseline has allowed us to search for genetic variants influencing MBD risk.

\section{METHODS}

Patients

We studied four independent cohorts of MM patients that had been the subject of previous GWAS $^{8-11}$ (Supplementary Figure 1): (i) My9 comprising 1205 MM cases from the UK Medical Research Council Myeloma-IX trial' ${ }^{12}$ (ISRCTN68454111); (ii) My11 comprising $768 \mathrm{MM}$ cases from the UK Medical Research Council Myeloma-IX trial $^{8}$ (ISRCTN49407852); (iii) HdB, comprising $1182 \mathrm{MM}$ patients recruited by the German-speaking Myeloma Multicentre Group (GMMG), coordinated by the University Clinic, Heidelberg $^{9}$ (ISRCTN644552890, ISRCTN05745813); (iv) ArK, comprising 619 newly diagnosed MM patients treated at the UAMS Myeloma Institute, Little Rock, AR, USA ${ }^{11}$ (NCT00580372, NCT00081939, NCT00572169, NCT00734877). The clinical characteristics and demographics of the patients in each of the four patient cohorts are summarized in Table 1. There was a higher proportion of patients with WHO performance stage $\geqslant 3 \mathrm{MM}$ in My9 and My11, reflecting in part the increased age of patients in the non-intensive arms of these trials. The study was approved by the respective institutional ethical review boards: MREC 02/8/95 (My9); MREC 17/09/09 (My11); 229/2003, S-337/2009, AFmu-119/2010 (HdB) and all participants provided written informed consent.

\section{Radiological assessment of bone lesions}

MBD was detected using axial survey in My9 and My11, axial skeletal survey (2001-2010) and whole body computed tomography (2011 onwards) in $\mathrm{HdB}$ and combined skeletal survey and skeletal computed tomography in ArK. The frequency of MBD was marginally higher in the HdB cohort $(P=0.01)$. Owing to the differences in sensitivity of the radiological methods used to detect $M B D$, patients were classified as either affected (MBD) or unaffected (no MBD). Age was not significantly associated with MBD in any of the four cohorts (that is, $P>0.05$ ).

\footnotetext{
'Division of Molecular Pathology, The Institute of Cancer Research, London, UK; ${ }^{2}$ Myeloma Institute, University of Arkansas for Medical Sciences, Little Rock, AR, USA; ${ }^{3}$ Department of Internal Medicine V, University of Heidelberg, Heidelberg, Germany; ${ }^{4}$ Division of Genetics and Epidemiology, The Institute of Cancer Research, London, UK; ${ }^{5}$ German Cancer Research Center, Heidelberg, Germany; ${ }^{6}$ Center for Primary Health Care Research, Lund University, Malmö, Sweden; ${ }^{7}$ Leeds Institute of Molecular Medicine, Section of Clinical Trials Research, University of Leeds, Leeds, UK; ${ }^{8}$ Department of Haematology, Newcastle University, Newcastle-Upon-Tyne, UK; ${ }^{9}$ Institute of Human Genetics, University of Bonn, Bonn, Germany; ${ }^{10}$ Division of Medical Genetics, Department of Biomedicine, University of Basel, Basel, Switzerland; ${ }^{11}$ Department of Genomics, Life \& Brain Center, University of Bonn, Bonn, Germany and ${ }^{12}$ National Center of Tumor Diseases, Heidelberg, Germany. Correspondence: Professor RS Houlston, Division of Genetics and Epidemiology, The Institute of Cancer Research, 15 Cotswold Road, Sutton SM2 5NG London, UK.
}

E-mail: richard.houlston@icr.ac.uk

Received 3 August 2015; revised 25 November 2015; accepted 30 November 2015; accepted article preview online 16 December 2015; advance online publication, 12 January 2016 
Table 1. Clinical characteristics and demographics of patients

\begin{tabular}{|c|c|c|c|c|}
\hline & My9 & My11 & $H d B$ & ArK \\
\hline Number of cases & 1205 & 768 & 1182 & 619 \\
\hline $\begin{array}{l}\text { Median age at } \\
\text { MM diagnosis }\end{array}$ & 64 & 66 & 57 & 59 \\
\hline \multicolumn{5}{|l|}{ Gender } \\
\hline Male & 718 (59.6\%) & $446(58.1 \%)$ & 699 (59.1\%) & $389(62.8 \%)$ \\
\hline Female & 487 (40.4\%) & $322(41.9 \%)$ & $483(40.9 \%)$ & $230(37.2 \%)$ \\
\hline \multicolumn{5}{|l|}{ ISS } \\
\hline 1 & $236(20.8 \%)$ & $181(24.9 \%)$ & $208(44.2 \%)$ & $293(47.3 \%)$ \\
\hline II & $457(40.3 \%)$ & 290 (39.8\%) & $160(34.0 \%)$ & $182(29.4 \%)$ \\
\hline III & $440(38.8 \%)$ & $257(35.3 \%)$ & $103(21.9 \%)$ & $144(23.3 \%)$ \\
\hline NA & 72 & 40 & 711 & 0 \\
\hline \multicolumn{5}{|c|}{ WHO performance stage } \\
\hline 0 & $299(25.0 \%)$ & $265(35.1 \%)$ & $181(48.1 \%)$ & NA \\
\hline 1 & 549 (45.9\%) & $319(42.3 \%)$ & $161(42.8 \%)$ & NA \\
\hline 2 & $224(18.7 \%)$ & $130(17.2 \%)$ & $28(7.4 \%)$ & NA \\
\hline$\geqslant 3$ & $125(10.4 \%)$ & $40(5.3 \%)$ & $6(1.6 \%)$ & NA \\
\hline $\mathrm{NA}$ & 8 & 14 & 806 & NA \\
\hline \multicolumn{5}{|l|}{ Bone disease } \\
\hline Yes & $875(72.6 \%)$ & $554(72.1 \%)$ & 912 (77.2\%) & $457(73.8 \%)$ \\
\hline No & $330(27.4 \%)$ & $214(27.9 \%)$ & $270(22.8 \%)$ & $162(26.2 \%)$ \\
\hline \multicolumn{5}{|l|}{ Vertebral fractures } \\
\hline Yes & 372 (41.3\%) & 222 (41.6\%) & NA & NA \\
\hline No & $528(58.7 \%)$ & 312 (58.4\%) & NA & NA \\
\hline NA & 305 & 234 & NA & NA \\
\hline \multicolumn{5}{|c|}{ Heavy chain paraprotein } \\
\hline $\operatorname{lgG}$ & $629(62.2 \%)$ & $403(56.1 \%)$ & $333(58.7 \%)$ & $353(57.1 \%)$ \\
\hline $\lg A$ & $224(22.1 \%)$ & $195(27.2 \%)$ & $128(22.6 \%)$ & $140(22.7 \%)$ \\
\hline $\lg D$ & $22(2.2 \%)$ & $13(1.8 \%)$ & $4(0.7 \%)$ & $4(0.6 \%)$ \\
\hline LCO & $130(12.8 \%)$ & $103(14.3 \%)$ & $94(16.6 \%)$ & $111(18.1 \%)$ \\
\hline $\begin{array}{l}\text { No } \\
\text { paraprotein }\end{array}$ & $7(0.7 \%)$ & $4(0.6 \%)$ & $8(1.4 \%)$ & $10(1.6 \%)$ \\
\hline NA & 193 & 50 & 615 & 1 \\
\hline \multicolumn{5}{|c|}{ Light chain paraprotein } \\
\hline Lambda & $352(34.7 \%)$ & 239 (33.2\%) & $187(33.1 \%)$ & $238(39.0 \%)$ \\
\hline Kappa & $662(65.3 \%)$ & $477(66.3 \%)$ & $378(66.9 \%)$ & $367(60.0 \%)$ \\
\hline No light chain & 0 & $3(0.4 \%)$ & 0 & $6(1.0 \%)$ \\
\hline NA & 191 & 49 & 617 & 8 \\
\hline \multicolumn{5}{|l|}{ Hyperdiploidy } \\
\hline Yes & 367 (55.6\%) & $222(48.9 \%)$ & 536 (55.9\%) & NA \\
\hline No & 293 (44.4\%) & $232(51.1 \%)$ & $423(44.1 \%)$ & NA \\
\hline NA & 545 & 314 & 223 & NA \\
\hline
\end{tabular}

Abbreviations: ISS, international staging system; LCO, light chain only; MM, multiple myeloma; NA, not available.

\section{Tumor karyotyping}

Conventional cytogenetics of tumors was conducted using standard karotyping methodologies, and standard criteria for the definition of a clone were applied. ${ }^{13}$ Fluorescent in situ hybridization and ploidy classification of My9 and My11 samples were conducted using the methods described by Chiecchio et al. ${ }^{14}$ Fluorescent in situ hybridization analysis and ploidy classification of $\mathrm{HdB}$ samples were performed as previously described. ${ }^{15}$ The XL IGH Break Apart probe (MetaSystems, Altlussheim, Germany) was used to detect immunoglobulin $\mathrm{H}$ translocations in $\mathrm{HdB}$ samples.

\section{Genotyping and quality control}

All cases were genotyped using Illumina Human OmniExpress arrays adhering to the manufacturer's protocols (Illumina, San Diego, CA, USA). Standard quality control was performed on all scans, excluding individuals with low call rate $(<90 \%)$ and extremely high or low heterozygosity $\left(P<1.0 \times 10^{-4}\right)$, as well as all individuals shown to be of non-European ancestry (using the HapMap version 2 CEU, JPT/CHB and YRI populations as a reference; Supplementary Figure 2). A summary of the number of genotyped single-nucleotide polymorphisms (SNPs) and the number of SNPs passing quality controls is shown in Supplementary Figure 1.

\section{Imputation}

Genotypes for common variants across the genome were imputed using data from 1000 Genomes Project (phase 1 integrated release 3, March
2012) and UK10K as reference in conjunction with IMPUTE2 v2.1.1 ${ }^{16}$ after pre-phasing with SHAPEIT software; ${ }^{17}$ poorly imputed SNPs defined by an information measure $<0.90$ were excluded. All genomic locations are given in National Center for Biotechnology Information Build 37/UCSC hg19 coordinates. All SNPs having a minor allele frequency $<1 \%$ were excluded.

\section{Statistical analysis}

We compared the relationship between genotype and presence or absence of MBD by logistic regression including covariates found in univariate analysis to influence MBD. We adjusted for the method of radiological assessment in each cohort and used eigenvalues in the analysis of the $\mathrm{HdB}$ series, to adjust for population substructure. $P$-values presented correspond to the significance of a test difference among all three of the genotype groups (common allele homozygote, heterozygote and rare allele homozygote). We confined our analysis to SNPs with a minor allele frequency $>1 \%$ because of extreme value of the test statistics. Overall statistical significance tests for each SNP were performed by combining the results for each cohort by a fixed-effects meta-analysis. All statistical tests were two-sided. Inflation of the test statistics, $\lambda$, was estimated by dividing the 45 th percentile of the test statistic by $0.357-$ the 45th percentile for a $X^{2}$ distribution on 1 degree of freedom. Betweenstudy heterogeneity was quantified using the $I^{2}$ statistic. Associations were regarded as statistically significant at a $P$-value $\leqslant 5.0 \times 10^{-8}$ (that is, genome-wide significance). All statistical analyses were performed using PLINK v1.07 ${ }^{18}$ and R (v3.1.3) software. ${ }^{19}$

\section{Functional prediction}

To explore the epigenetic profile of genomic location associated with MBD, we used ENCODE histone modification data and HaploReg and RegulomeDB ${ }^{20,21}$ to examine whether any of the SNPs or their proxies (that is, $r^{2}>0.8$ in the 1000 Genomes European reference panel) annotate transcription factor binding or enhancer elements. We assessed sequence conservation using Genomic Evolutionary Rate Profiling scores. ${ }^{22}$

\section{RESULTS}

Relationship between genotype and bone lesions

After quality control measures were applied, genotype data on 5531610 SNPs was available for 3774 MM cases with MBD data. Quantile-Quantile plots of test statistics of the relationship between SNP genotype and MBD for each of the four cohorts is shown in Supplementary Figure 3; inflation factors $\lambda=1.002-1.01$, $\lambda=1.0008$ for the meta-analysis (Supplementary Figure 4).

Nine SNPs showed an association with MBD and reached genome-wide significance (Figures 1 and 2, Supplementary Table 1). All nine SNPs were located in the same region at $8 q 24.12$ and were in strong linkage disequilibrium (LD). The strongest association at $8 \mathrm{q} 24.12$ was provided by the common SNP rs4407910 (risk allele frequency $=0.50$, odds ratio $=1.38,95 \%$ confidence interval $=1.24-1.54, P=4.02 \times 10^{-9}$ ). The association was consistent across each of the four patient cohorts (Figure 3 and there was no significant between-study heterogeneity $\left(P_{\text {het }}=0.44, I^{2}=0 \%\right)$. An increased prevalence of MBD has been observed in male patients and hyperdiploid $\mathrm{MM}^{23,24}$ Stratifying data by sex or ploidy did not provide evidence for a differential effect of rs 4407910 genotypes on the risk of MBD (Supplementary Figures 5 and 6). Variation at $8 q 24.12$ has been previously associated with BMD and osteoporosis, but not as strongly with risk of fracture. ${ }^{25-28}$ Meta-analysis of My9 and My11 data showed no evidence that rs4407910 genotype influenced the risk of vertebral body fracture $(P=0.28)$.

The MBD risk SNP rs4407910 localizes $19 \mathrm{~Kb} 3^{\prime}$ to the gene encoding TNFRSF11B (tumor necrosis factor receptor superfamily, member $11 \mathrm{~b}$; alias osteoprotegerin; $O P G$ ). The genomic region contains multiple enhancer markers from several tissue types including bone marrow cells (Supplementary Table 2 and Supplementary Figure 7). In lymphoblast and other tissues, rs4355801, which is in perfect LD with rs4407910 $\left(r^{2}=1.0\right.$, 
$\left.D^{\prime}=1.0\right)$ is associated with $O P G$ expression; the risk allele for MBD being associated with reduced OPG expression (Supplementary Table 3). ${ }^{29-32}$

In addition to rs4407910, we detected a promising association for MBD marked by rs74676832 at $19 q 13.43$ (odds ratio $=1.97$, $95 \%$ confidence interval $=1.50-2.59, P=9.33 \times 10^{-7}, P_{\text {het }}=0.43$, $P^{2}=0 \%$ ). rs 74676832 is located within a $29 \mathrm{~kb}$ region of LD intergenic to ZNF444 and GALP (Figures 1 and 3 and Supplementary Figure 8).

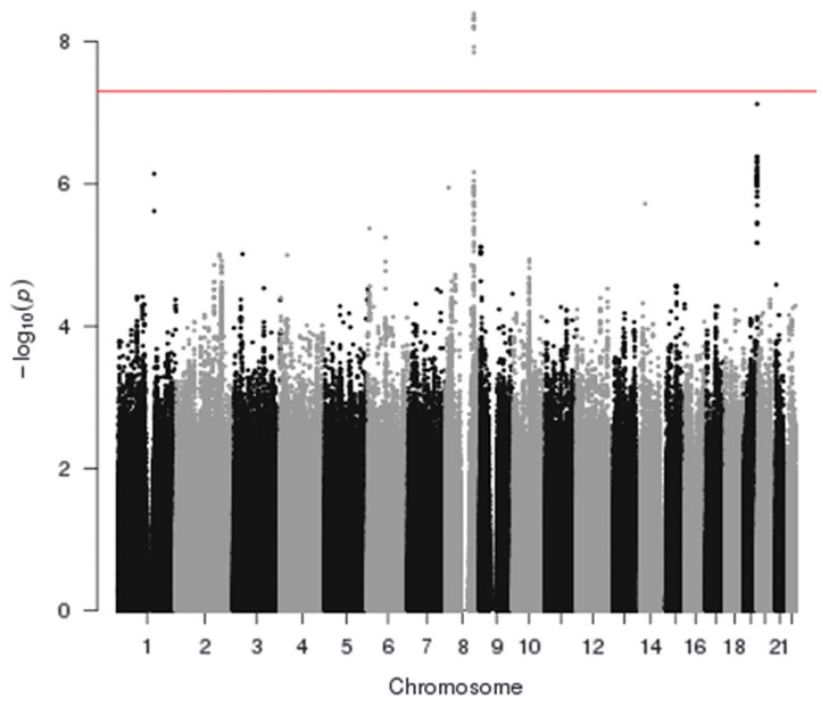

Figure 1. Association plot for combined analyses. The $P$-values of the association between each single-nucleotide polymorphism (SNP) and MBD. The $y$ axis shows the $-\log _{10} P$-values of each SNP analyzed, and the $x$ axis shows their respective chromosome position. The red horizontal line corresponds to $P=5.0 \times 10^{-8}$. All statistical tests were two-sided.
Impact of alleles influencing BMD on MM bone disease

Variation at 8q24.12 marked by rs23062375 and rs11995824, which are intronic SNPs in OPG and in LD with rs4407910 (respective LD metrics $r^{2}$ and $D^{\prime}-0.78,0.93$ and $0.79,1.0$ ) have been demonstrated to influence BMD..$^{25-27,31,33}$ To explore the possibility that other genetic variants influencing BMD also influence MBD, we investigated the association at 77 established risk loci for BMD with MBD $^{25-28}$ (Supplementary Table 4). Aside from the $8 \mathrm{q} 24.12$ SNPs, no other BMD locus showed an association with MBD after adjusting for multiple testing (that is, $P>0.001$ ). Moreover, there was no over-representation of association for MBD across the 77 SNPs (Supplementary Table 5).

\section{DISCUSSION}

Our findings support the hypothesis that an individual's risk of developing MBD is influenced by germline variation. Specifically, we identified a locus at 8q24.12 (rs4407910) associated with MBD. rs4407910 maps to a region of LD that only contains OPG, and when taken in conjunction with eQTL data it is likely that reduced OPG expression is the functional basis of the $8 q 24.12$ association. ${ }^{31}$

Osteoclasts (OCs) are bone-resorptive cells, which are critical for the integrity of bone. OC-differentiation and activation is dependent on activation of nuclear factor-kB ligand (RANKL) signaling through the p38 MAPK pathway. ${ }^{34-36}$ OPG is a negative regulator of bone resorption acting as a decoy receptor for RANKL, decreasing bone resorption through inhibiting differentiation of OC precursors, activating mature $\mathrm{OCs}$ and stimulating $\mathrm{OC}$ apoptosis. Germline inactivating mutations in $O P G$ are responsible for the autosomal dominant diseases: early-onset and familial Paget'disease, familial expansile osteolysis and expansile skeletal hyperphosphatasia, which are characterised by the development of expansile osteolytic bone lesions. ${ }^{37,38}$ Myeloma cells express RANKL and treatment of mice models of MM with OPG has been demonstrated to prevent lytic bone lesions, maintaining cancellous bone volume and inhibiting OC formation. ${ }^{12,32}$

Current clinical management of MBD involves reducing myeloma cell infiltration of the bone marrow using

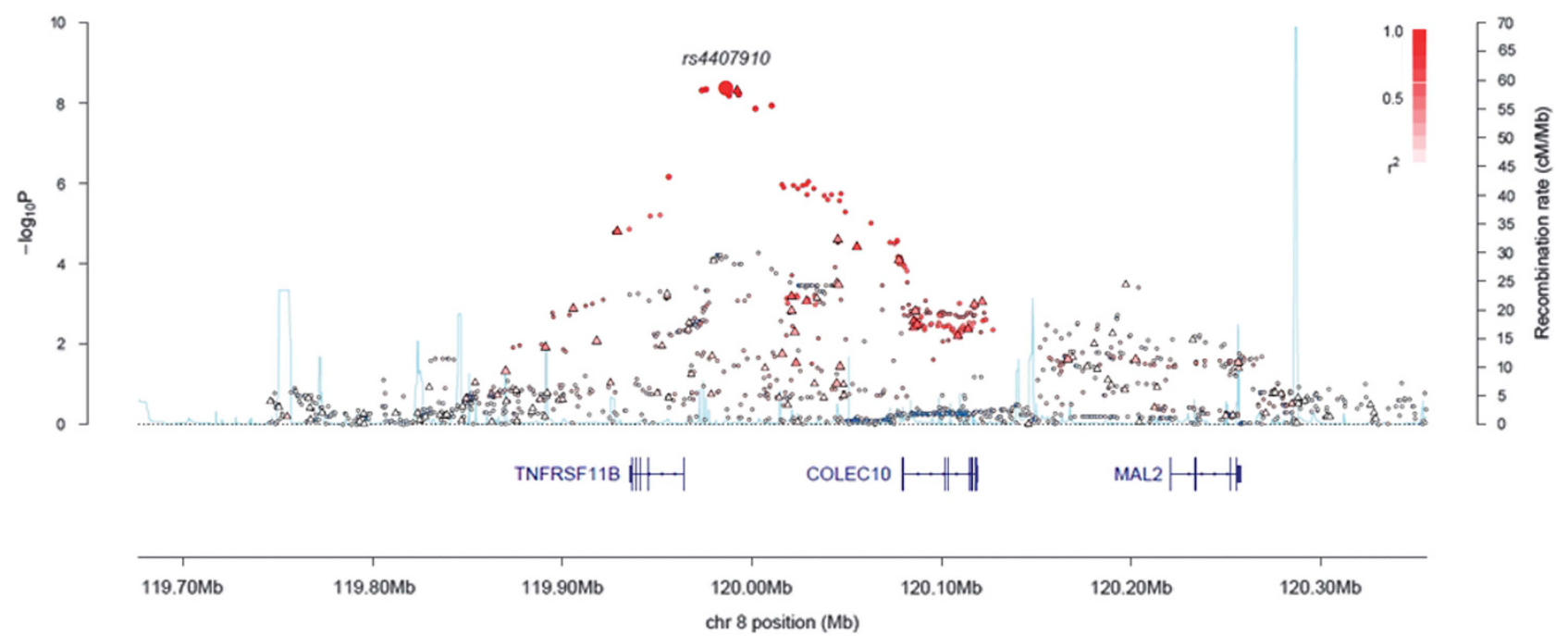

Figure 2. Regional plot of association and recombination rates for the $8 q 24.12$ locus. Plots show association results of both genotyped (triangles) and imputed (circles) SNPs and recombination rates. $-\log _{10} P$-values ( $y$ axes) of the SNPs are shown according to their chromosomal positions ( $x$ axes). rs4407910 shown as a large diamond. The color intensity of each symbol reflects the extent of LD with rs4407910 white $\left(r^{2}=0\right)$ through to dark red $\left(r^{2}=1.0\right)$. Genetic recombination rates, estimated using HapMap samples from Utah residents of western and northern European ancestry (CEU), are shown with a light blue line. Physical positions are based on NCBI build 37 of the human genome. Also shown are the relative positions of genes and transcripts mapping to the region of association. 


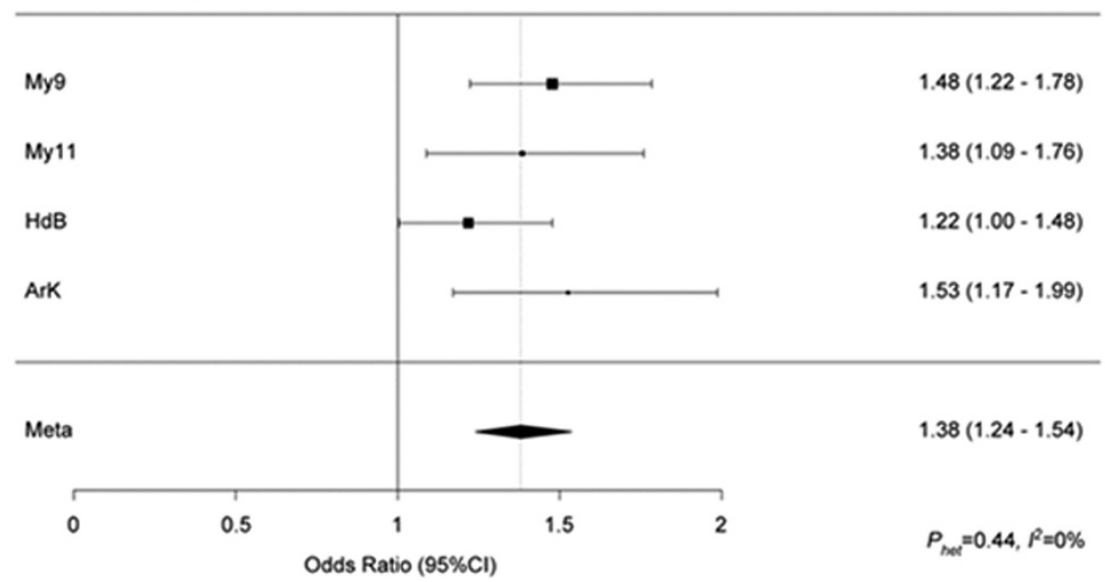

b

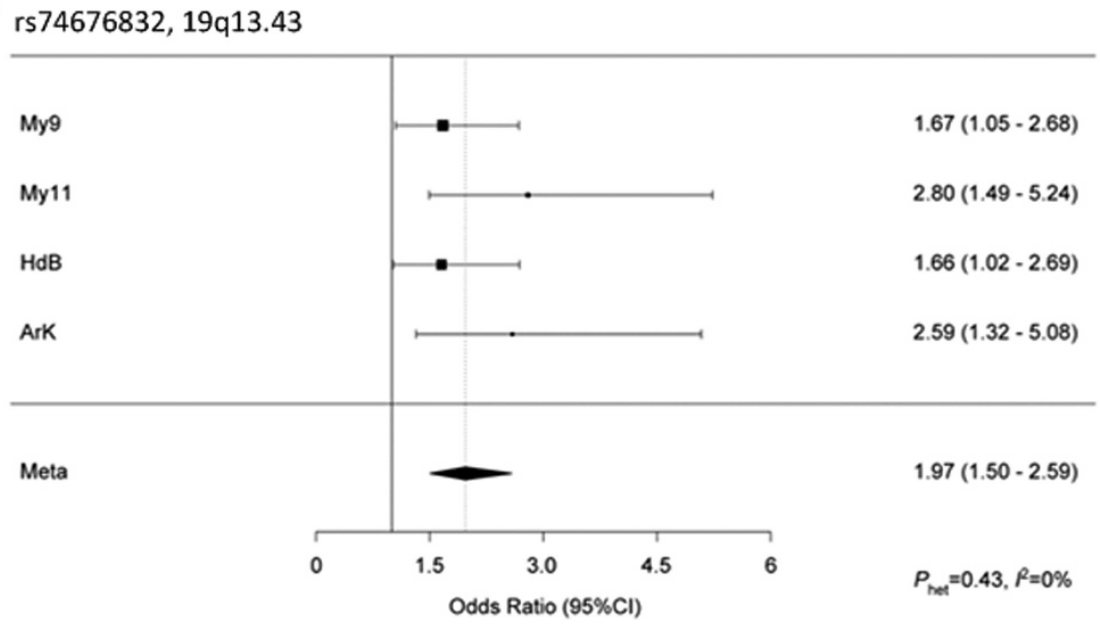

Figure 3. Forest plot of the ORs for the association between (a) rs4407910, (b) rs74676832 and MBD. Studies were weighted according to the inverse of the variance of the log of the OR calculated. Horizontal lines: $95 \% \mathrm{Cl}$. Box: OR point estimate; box area is proportional to the weight of the study. Diamond (and broken line): overall summary estimate, with $\mathrm{Cl}$ given by its width. Unbroken vertical line: null value (OR $=1.0)$.

bisphosphonates to inhibit OC activity; however, there remains a need to develop more targeted treatments. Established MM therapies such as immunomodulatory drugs and proteasome inhibitors partially exert their activity through the OPG/RANK/ RANKL system. ${ }^{39,40}$ Hence, targeting the OPG/RANK/RANKL system through specific agents such as raloxifene may have therapeutic potential. ${ }^{41,42}$ Raising OPG levels directly by infusion of recombinant OPG (Fc-OPG) suppresses bone resorption. ${ }^{43}$ Potential concerns over the generation of anti-Fc-OPG and binding to TRAIL has however shifted further development away from Fc-OPG as a RANK inhibitor, to Denosumab, a human monoclonal antibody against RANKL. ${ }^{44,45}$

In addition to the $8 \mathrm{q} 24.12$ locus we identified a promising signal at 19q13.43 proximal to genes ZNF444 and GALP. GALP encodes a member of the galanin family of neuropeptides that are active within the central nervous system. ${ }^{46}$ ZNF444, a zinc finger protein activates a scavenger receptor gene, which participates in the degradation of acetylated low-density lipoprotein. ${ }^{47}$ Intriguingly, acetylated low-density lipoprotein promotes osteoblastic differentiation, making ZNF444 a credible candidate gene for the basis of the $19 q 13.43$ MBD association. ${ }^{48}$

In making these conclusions, we have made use of various differing methods for MDB, which have different sensitivity for its detecting, the differences are between, not within, cohorts and hence systematic basis is unlikely to have impacted on our findings. In addition, the association was seen in each of the four patient cohorts and was not confined to a specific MM subtype. It is important to note, that the frequency of no MBD is equivalent in each of the four case series.

Bone loss in $M M$ is unlikely to be exclusively attributable to RANKL/RANK/OPG providing a rationale for conducting further GWAS-based analyses to identify additional MBD risk variants. It is, however, noteworthy that SNPs, other than rs4407910, which are strongly associated with BMD, were not found by us to be associated with MBD. As our power to detect an allele with an odds ratio $>1.5$ was high ( $>90 \%$ power if the minor allele frequency $>0.2$ ), it implies that few such alleles are likely to exist. However our power to detect an association for an odds ratio $~ 1.2$ is poor (that is, $<20 \%$ ), and such variants could readily exist. It is, therefore, apparent that larger studies will be required to identify additional risk loci for MBD. Although the impact of any individual genetic variant discovered by GWAS influencing MBD may be relatively modest, their identification serves to highlight genes and pathways relevant to developing novel intervention strategies. The genotyping of patients from ongoing and future clinical trials is likely to be especially informative in establishing the relationship between markers of MBD and specific therapies.

In summary, our observations provide the first evidence that germline variation influences a MM patient's risk of developing MBD and importantly impacts on this throughout disease 
progression. These results also provide further support for the importance of the RANK/RANKL/OPG pathway in the development of MBD. As no other established BMD locus showed an association with $M B D$, our results suggest the interaction between myeloma cells and bone remodeling is primarily dictated by this pathway. These findings will contribute to the development of future strategies for prevention of MBD by defining those MM patients at high risk of MBD and who may maximally benefit from therapeutic intervention.

\section{CONFLICT OF INTEREST}

GHJ has received honoraria from Celgene and Janssen-Cilag for speaking at educational meetings. GJM has participated in advisory boards for, received payment for lectures and development of educational presentations from, and has received travel support from Celgene, Novartis, Merck and Johnson \& Johnson. FED has participated in advisory boards and spoken at meetings for Celgene, Ortho Biotech and Novartis, and has received travel support to attend meetings from Celgene and Ortho Biotech. JH has participated in advisory boards for Novartis, and has received travel support to attend meetings from Celgene, Janssen-Cilag and Takeda. HG has participated in advisory boards for, received payment for lectures and development of educational presentations from, and has received travel support from Celgene, Jassen-Cilag, Chugai, Novartis, Bristol-Myers Squibb, Millenium, Onyx, Amgen and Takeda. The remaining authors declare no conflict of interest.

\section{ACKNOWLEDGEMENTS}

Myeloma UK provided principal funding for this study in the United Kingdom. Additional funding was provided by Leukaemia Lymphoma Research, Cancer Research UK (C1298/A8362 supported by the Bobby Moore Fund) and the NHS via the Biological Research Centre of the National Institute for Health Research at the Royal Marsden Hospital NHS Trust. The UAMS GWAS was supported by a grant from the National Institutes of Health (P01CA055819). In Germany, funding was provided to Dietmar-Hopp-Stiftung Walldorf, Deutsche Krebshilfe, the University Hospital Heidelberg and Multiple Myeloma Research Foundation. Additionally, funding from by the German Ministry of Education and Science (Cliommics 01ZX1309B) and the German Research Council (DFG; Project SI 236/8-1, SI236/9-1, ER 155/6-1). We are extremely grateful to all investigators who contributed to the generation of this data set. Finally, we are grateful to all the patients and investigators at the individual centers for their participation. We also thank the staff of the CTRU University of Leeds and the NCRI haematology Clinical Studies Group.

\section{AUTHOR CONTRIBUTIONS}

Conception and design: GJM, KH, HG, DCJ, RSH. Provision of study materials or patients: GJM, FED, BB, JH, UB, HG, MK, GHJ. Collection and assembly of data: $\mathrm{PH}, \mathrm{MMN}, \mathrm{NW}, \mathrm{JN}, \mathrm{OWS}, \mathrm{JH}, \mathrm{DC}, \mathrm{WAG}$. Data analysis and interpretation: $\mathrm{BC}, \mathrm{AF}$, NW, JM, DCJ, RSH. Manuscript writing: GJM, KH, HG, DCJ, RSH. Final approval of manuscript: BC, AF, KH, NW, HG, PH, MMN, JH, UB, BB, FED, GJM, OWS, DC, WAG, $J N, G H J, M F, J M, D C J, R S H$.

\section{REFERENCES}

1 Kyle RA, Rajkumar SV. Multiple myeloma. N Engl J Med 2004; 351: 1860-1873.

2 Palumbo A, Anderson K. Multiple myeloma. N Engl J Med 2011; 364: 1046-1060.

3 Saad F, Lipton A, Cook R, Chen YM, Smith M, Coleman R. Pathologic fractures correlate with reduced survival in patients with malignant bone disease. Cancer 2007; 110: 1860-1867.

4 Giuliani N, Rizzoli V, Roodman GD. Multiple myeloma bone disease: pathophysiology of osteoblast inhibition. Blood 2006; 108: 3992-3996.

5 Greipp PR, San Miguel J, Durie BG, Crowley JJ, Barlogie B, Blade J et al. International staging system for multiple myeloma. J Clin Oncol 2005; 23: 3412-3420.

6 Krall EA, Dawson-Hughes B. Heritable and life-style determinants of bone mineral density. J Bone Miner Res 1993; 8: 1-9.

7 Richards JB, Zheng HF, Spector TD. Genetics of osteoporosis from genome-wide association studies: advances and challenges. Nat Rev Genet 2012; 13: 576-588.

8 Chubb D, Weinhold N, Broderick P, Chen B, Johnson DC, Forsti A et al. Common variation at $3 q 26.2,6 p 21.33,17 p 11.2$ and $22 q 13.1$ influences multiple myeloma risk. Nat Genet 2013; 45: 1221-1225.

9 Weinhold N, Johnson DC, Chubb D, Chen B, Forsti A, Hosking FJ et al. The CCND1 c.870G $>$ A polymorphism is a risk factor for $\mathrm{t}(11 ; 14)(\mathrm{q} 13 ; \mathrm{q} 32)$ multiple myeloma. Nat Genet 2013; 45: 522-525.
10 Broderick P, Chubb D, Johnson DC, Weinhold N, Forsti A, Lloyd A et al. Common variation at 3 p22.1 and 7 p15.3 influences multiple myeloma risk. Nat Genet 2012; 44: 58-61.

11 Erickson SW, Raj VR, Stephens OW, Dhakal I, Chavan SS, Sanathkumar N et al. Genome-wide scan identifies variant in 2 q12.3 associated with risk for multiple myeloma. Blood 2014; 124: 2001-2003.

12 Croucher PI, Shipman CM, Lippitt J, Perry M, Asosingh K, Hijzen A et al. Osteoprotegerin inhibits the development of osteolytic bone disease in multiple myeloma. Blood 2001; 98: 3534-3540.

13 Fonseca R, Barlogie B, Bataille R, Bastard C, Bergsagel PL, Chesi M et al. Genetics and cytogenetics of multiple myeloma: a workshop report. Cancer Res 2004; 64: 1546-1558.

14 Chiecchio L, Protheroe RK, Ibrahim AH, Cheung KL, Rudduck C, Dagrada GP et al. Deletion of chromosome 13 detected by conventional cytogenetics is a critical prognostic factor in myeloma. Leukemia 2006; 20: 1610-1617.

15 Neben K, Jauch A, Bertsch U, Heiss C, Hielscher T, Seckinger A et al. Combining information regarding chromosomal aberrations $t(4 ; 14)$ and del(17p13) with the International Staging System classification allows stratification of myeloma patients undergoing autologous stem cell transplantation. Haematologica 2010; 95: 1150-1157.

16 Howie BN, Donnelly P, Marchini J. A flexible and accurate genotype imputation method for the next generation of genome-wide association studies. PLoS Genet 2009; 5: e1000529.

17 Delaneau O, Zagury JF, Marchini J. Improved whole-chromosome phasing for disease and population genetic studies. Nat Methods 2013; 10: 5-6.

18 Purcell S, Neale B, Todd-Brown K, Thomas L, Ferreira MA, Bender D et al. PLINK: a tool set for whole-genome association and population-based linkage analyses. Am J Hum Genet 2007; 81: 559-575.

19 R-Core-Team. R. A Language and Environment for Statistical Computing. R Foundation for Statistical Computing: Vienna, Austria, ISBN 3-900051-07-0, URL http://www.R-project.org/, 2013.

20 Boyle AP, Hong EL, Hariharan M, Cheng Y, Schaub MA, Kasowski M et al. Annotation of functional variation in personal genomes using RegulomeDB. Genome Res 2012; 22: 1790-1797.

21 Ward LD, Kellis M. HaploReg: a resource for exploring chromatin states, conservation, and regulatory motif alterations within sets of genetically linked variants. Nucleic Acids Res 2012; 40: D930-D934.

22 Cooper GM, Goode DL, Ng SB, Sidow A, Bamshad MJ, Shendure J et al. Single-nucleotide evolutionary constraint scores highlight disease-causing mutations. Nat Methods 2010; 7: 250-251.

23 Chng WJ, Santana-Davila R, Van Wier SA, Ahmann GJ, Jalal SM, Bergsagel PL et al. Prognostic factors for hyperdiploid-myeloma: effects of chromosome 13 deletions and $\mathrm{lgH}$ translocations. Leukemia 2006; 20: 807-813.

24 Wu P, Walker BA, Brewer D, Gregory WM, Ashcroft J, Ross FM et al. A gene expression-based predictor for myeloma patients at high risk of developing bone disease on bisphosphonate treatment. Clin Cancer Res 2011; 17: 6347-6355.

25 Styrkarsdottir U, Halldorsson BV, Gretarsdottir S, Gudbjartsson DF, Walters GB, Ingvarsson $T$ et al. Multiple genetic loci for bone mineral density and fractures. N Engl J Med 2008; 358: 2355-2365.

26 Kemp JP, Medina-Gomez C, Estrada K St, Pourcain B, Heppe DH, Warrington NM et al. Phenotypic dissection of bone mineral density reveals skeletal site specificity and facilitates the identification of novel loci in the genetic regulation of bone mass attainment. PLoS Genet 2014; 10: e1004423.

27 Estrada K, Styrkarsdottir U, Evangelou E, Hsu YH, Duncan EL, Ntzani EE et al. Genome-wide meta-analysis identifies 56 bone mineral density loci and reveals 14 loci associated with risk of fracture. Nat Genet 2012; 44: 491-501.

28 Paternoster L, Lorentzon M, Lehtimaki T, Eriksson J, Kahonen M, Raitakari O et al. Genetic determinants of trabecular and cortical volumetric bone mineral densities and bone microstructure. PLoS Genet 2013; 9: e1003247.

29 Liang L, Morar N, Dixon AL, Lathrop GM, Abecasis GR, Moffatt MF et al. A crossplatform analysis of 14,177 expression quantitative trait loci derived from lymphoblastoid cell lines. Genome Res 2013; 23: 716-726.

30 Grundberg E, Small KS, Hedman AK, Nica AC, Buil A, Keildson S et al. Mapping cisand trans-regulatory effects across multiple tissues in twins. Nat Genet 2012; 44: 1084-1089.

31 Richards JB, Rivadeneira F, Inouye M, Pastinen TM, Soranzo N, Wilson SG et al. Bone mineral density, osteoporosis, and osteoporotic fractures: a genome-wide association study. Lancet 2008; 371: 1505-1512.

32 Rabin N, Kyriakou C, Coulton L, Gallagher OM, Buckle C, Benjamin R et al. A new xenograft model of myeloma bone disease demonstrating the efficacy of human mesenchymal stem cells expressing osteoprotegerin by lentiviral gene transfer. Leukemia 2007; 21: 2181-2191.

33 Rivadeneira F, Styrkarsdottir U, Estrada K, Halldorsson BV, Hsu YH, Richards JB et al. Twenty bone-mineral-density loci identified by large-scale meta-analysis of genome-wide association studies. Nat Genet 2009; 41: 1199-1206. 
34 Simonet WS, Lacey DL, Dunstan CR, Kelley M, Chang MS, Luthy R et al. Osteoprotegerin: a novel secreted protein involved in the regulation of bone density. Cell 1997; 89: 309-319.

35 Yasuda H, Shima N, Nakagawa N, Mochizuki SI, Yano K, Fujise N et al. Identity of osteoclastogenesis inhibitory factor (OCIF) and osteoprotegerin (OPG): a mechanism by which OPG/OCIF inhibits osteoclastogenesis in vitro. Endocrinology 1998; 139: 1329-1337.

36 Sezer O, Heider U, Zavrski I, Kuhne CA, Hofbauer LC. RANK ligand and osteoprotegerin in myeloma bone disease. Blood 2003; 101: 2094-2098.

37 Whyte MP, Obrecht SE, Finnegan PM, Jones JL, Podgornik MN, McAlister WH et al. Osteoprotegerin deficiency and juvenile Paget's disease. N Engl J Med 2002; 347: 175-184.

38 Ralston SH. Clinical practice. Paget's disease of bone. N Engl J Med 2013; 368: 644-650

39 Giuliani N, Morandi F, Tagliaferri S, Lazzaretti M, Bonomini S, Crugnola M et al. The proteasome inhibitor bortezomib affects osteoblast differentiation in vitro and in vivo in multiple myeloma patients. Blood 2007; 110: 334-338.

40 Bolzoni M, Storti P, Bonomini S, Todoerti K, Guasco D, Toscani D et al. Immunomodulatory drugs lenalidomide and pomalidomide inhibit multiple myelomainduced osteoclast formation and the RANKL/OPG ratio in the myeloma microenvironment targeting the expression of adhesion molecules. Exp Hematol 2013; 41: 387-397 e381.

41 Olivier S, Close P, Castermans E, de Leval L, Tabruyn S, Chariot A et al. Raloxifene-induced myeloma cell apoptosis: a study of nuclear factor-kappaB inhibition and gene expression signature. Mol Pharmacol 2006; 69: 1615-1623.

42 Sola B, Renoir JM. Estrogenic or antiestrogenic therapies for multiple myeloma? Mol Cancer 2007; 6: 59.

43 Body JJ, Greipp P, Coleman RE, Facon T, Geurs F, Fermand JP et al. A phase I study of AMGN-0007, a recombinant osteoprotegerin construct, in patients with multiple myeloma or breast carcinoma related bone metastases. Cancer 2003; 97: 887-892.

44 Reddy GK, Nadler E, Jain VK. Denosumab (AMG 162), a Fully Human Monoclonal Antibody Against RANK Ligand Activity. Support Cancer Ther 2005; 3: 14-15.

45 Bekker PJ, Holloway DL, Rasmussen AS, Murphy R, Martin SW, Leese PT et al. A single-dose placebo-controlled study of AMG 162, a fully human monoclonal antibody to RANKL, in postmenopausal women. J Bone Miner Res 2004; 19: 1059-1066.

46 Lang R, Gundlach AL, Kofler B. The galanin peptide family: receptor pharmacology, pleiotropic biological actions, and implications in health and disease. Pharmacol Ther 2007; 115: 177-207.

47 Adachi $\mathrm{H}$, Tsujimoto $\mathrm{M}$. Characterization of the human gene encoding the scavenger receptor expressed by endothelial cell and its regulation by a novel transcription factor, endothelial zinc finger protein-2. J Biol Chem 2002; 277: 24014-24021.

48 Proudfoot D, Davies JD, Skepper JN, Weissberg PL, Shanahan CM. Acetylated lowdensity lipoprotein stimulates human vascular smooth muscle cell calcification by promoting osteoblastic differentiation and inhibiting phagocytosis. Circulation 2002; 106: 3044-3050.

\section{(c) (i)}

This work is licensed under a Creative Commons Attribution 4.0 International License. The images or other third party material in this article are included in the article's Creative Commons license, unless indicated otherwise in the credit line; if the material is not included under the Creative Commons license, users will need to obtain permission from the license holder to reproduce the material. To view a copy of this license, visit http://creativecommons.org/licenses/ by/4.0/

Supplementary Information accompanies this paper on the Leukemia website (http://www.nature.com/leu) 